\title{
Naturaleza jurídica del contrato de concesión de obra por vía de asociaciones público-privadas: fortalezas y deficiencias*
}

\author{
Luis Carlos Guzmán Cuenca**
}

Recibido: 12 de octubre de 2018 - Aprobado: 18 de enero de 2019

\section{Resumen}

Las asociaciones público-privadas (APP) están reguladas por la Ley 1508/2012, de 10 de enero, en la que el privado asume la construcción, operación y administración de la infraestructura concesionada por el Estado. El propósito de este artículo es establecer cuáles son las fortalezas y las deficiencias del contrato de concesión de obra cuando se gestiona por vía de las APP. Se identificó que su principal fortaleza es que se le paga al contratista en la medida en que preste un servicio de calidad y eficiente en la infraestructura, y su mayor deficiencia es que solo se está aplicando en mayor medida en los contratos de concesión de obra de cuarta generación en el país. Asimismo, se determinó la naturaleza jurídica del contrato de concesión de obra bajo la modalidad de APP, se identificaron las fortalezas del contrato de concesión de obra en las APP, se establecieron las deficiencias del contrato de concesión de obra por vía de APP y se formularon propuestas o recomendaciones frente a los contratos de concesión de obra gestionadas por vía de las APP. La metodología utilizada para la elaboración del artículo fue la cualitativa, con el fin

* $\quad$ Este artículo es producto del proyecto de investigación “Naturaleza jurídica del contrato de concesión de obra por vía de asociaciones público-privadas (APP): fortalezas y deficiencias", gestionado en la Especialización en Derecho Administrativo de la Universidad Libre de Bogotá. Citar como: Guzmán Cuenca, L. C. (2019). Naturaleza jurídica del contrato de concesión de obra por vía de asociaciones público-privadas: fortalezas y deficiencias. Revista IUSTA, 51(2), 153-184. DOI: https://doi.org/10.15332/25005286.5034

** Abogado por la Fundación Universitaria Los Libertadores y especialista en Derecho Administrativo por la Universidad Libre Bogotá. Asesor jurídico de la Secretaría de Educación, Bogotá, Colombia. Orcid: https://orcid.org/0000-0002-0796-9303. Correo electrónico: luiscarlosguzmanc@gmail.com 
de abordar con más detalle y precisión las definiciones o los significados en cuanto al contrato de concesión de obra y sobre las APP. El método de investigación utilizado fue el dogmático, ya que se propuso analizar y describir la naturaleza jurídica de los contratos de concesión de obra gestionados por las APP, además de ayudar a establecer sus fortalezas, deficiencias, y con ellas convertirlas en unas posibles propuestas o recomendaciones a este tipo de contratación.

Palabras clave: asociaciones público-privadas, contrato de concesión de obra, infraestructura vial.

\title{
LEGAL NATURE OF THE WORK CONCESSION AGREEMENT THROUGH PUBLIC-PRIVATE PARTNERSHIPS: STRENGTHS AND DEFICIENCIES
}

\begin{abstract}
Public-private partnerships (PPPs) are regulated by Law 1508/2012, of January 10, in which the private sector assumes the construction, operation and administration of the infrastructure given in concession by the State. The purpose of this article is to establish the strengths and weaknesses of the work concession agreement when it is managed through PPPs. It was identified that its main strength is that the contractor is paid insofar as it provides a quality and efficient service in the infrastructure, and its biggest deficiency is that it is only being applied to a greater extent in the fourth generation works concession agreements in the country. Likewise, the legal nature of the work concession agreement under PPPs was determined, the strengths of the work concession agreement in the PPPs were identified, the deficiencies of the work concession agreement were established through PPPs and proposals or recommendations were formulated for the works concession agreements managed through the PPPs. The methodology used for the preparation of the article was qualitative, in order to address in more detail and accuracy the definitions or meanings regarding the concession agreement and on PPPs. The research method used was dogmatic, since it was proposed to analyze and describe the legal nature of the works concession agreements managed by PPPs, in addition to helping to establish their strengths, deficiencies, and converting them into possible proposals or recommendations to this type of contracting.
\end{abstract}

Keywords: public-private partnerships, work concession agreement, road infrastructure. 


\section{NATUREZA JURÍdica do CONTRATO DE CONCESSÃo DE OBRA POR VIA DE ASSOCIAÇÕES PÚBLICO-PRIVADAS: FORTALEZAS E DEFICIÊNCIAS}

\section{Resumo}

As associações público-privadas (APP) estão regulamentadas pela Lei 1508/2012, de 10 de janeiro, na qual o privado assume a construção, operação e administração da infraestrutura concedida pelo Estado. O propósito deste artigo é estabelecer quais são as fortalezas e as deficiências do contrato de concessão de obra quando é administrada pelas APP. Identificou-se que sua principal fortaleza é que se paga ao contratista na medida em que preste um serviço de qualidade e eficiente na infraestrutura e sua maior deficiência é que somente se está aplicando em maior medida nos contratos de concessão de obra de quarta geração no país. Igualmente, determinou-se a natureza jurídica do contrato de concessão de obra sob a modalidade de APP, identificaram-se as fortalezas do contrato de concessão de obra nas APP, estabeleceram-se as deficiências do contrato de concessão de obra por via de APP e formularam-se propostas ou recomendações perante aos contratos de concessão de obra administradas por via das APP. A metodologia utilizada para elaboração do artigo foi qualitativa, com o objetivo de abordar com mais detalhe e precisão nas definições ou significados no relacionado ao contrato de concessão de obra e sobre as APP. O método de pesquisa utilizado foi o dogmático, porque se propôs analisar e descrever natureza jurídica dos contratos de concessão de obra administrados pelas APP, além de ajudar a estabelecer suas fortalezas, deficiências e com estas convertê-las em umas possíveis propostas ou recomendações a este tipo de contratação.

Palavras-chave: associações público-privadas, contrato de concessão de obra, infraestrutura viária.

\section{Introducción}

En Colombia, en 1983, se empieza a regular una tipología contractual denominada contrato de concesión por medio del Decreto 222/1983, de 2 de febrero. Diez años más tarde se expide la Ley 80/1993, de 28 de octubre, por la cual se expide el Estatuto General de Contratación de la Administración Pública, en el que se 
evidencian dos tipos de contratos de concesión: uno de obra y otro destinado al servicio y uso público; el que nos atañe es el contrato de concesión de obra.

Ahora bien, desde aproximadamente 1993 hasta la actualidad, este tipo de contrato ha tenido inconvenientes o fallas, y unos de sus principales factores ha sido el incumplimiento por parte del concesionario en el momento de entregar la infraestructura a tiempo, además deficiencias en niveles de servicio, calidad y disponibilidad de la obra. Por tal razón, se aprueba la Ley 1508/2012, de 10 de enero.

Por eso, con este artículo, se busca establecer cuáles son las fortalezas y deficiencias del contrato de concesión de obra y porqué ha sido tan importante en el desarrollo o en la implementación en mayor medida en los contratos de concesión de cuarta generación, en el que el desarrollo de la infraestructura vial ha sido de forma acelerada cumpliendo con todos los estándares de calidad y servicio, de modo que ha sido todo un éxito en el país. Por tal razón, se realizó la presente investigación jurídica frente a esta nueva forma de contratación, que, aunque lleva seis años de vigencia, posee grandes beneficios para el Estado y su población.

Por esta razón, este artículo es relevante, no solo para la academia, sino para las entidades públicas representadas en sus funcionarios, a fin de que profundicen en este tipo de contratación entre el Estado y el sector privado, denominado contrato de concesión de obra por vía de asociaciones público-privadas (APP), y así apostarle a este tipo de contratación en el país y sacarle todo el provecho y las ventajas que nos brinda, para un desarrollo económico y social más satisfactorio en nuestra sociedad.

\section{Marco normativo de los contratos de concesión de obra por vía de las APP}

En este artículo, hablaremos del contrato de concesión de obra por vía de las APP en el país, pero, antes de esto, debemos tener claro que la contratación estatal en Colombia persigue satisfacer las necesidades de la población, en los fines del Estado, basado en sus principios de transparencia, economía, responsabilidad, selección objetiva, conmutatividad y equilibrio económico. Por consiguiente, la Ley 80/1993, de 28 de octubre, la Ley 1150/2007, de 16 de julio y la Ley 1882/2018, de 15 de enero, pretenden cumplir con todos los preceptos mencionados, según el principio del interés general sobre el interés individual (Ley 80/1993, de 28 de octubre, art. 3). 
Ahora bien, en la contratación estatal, existen diferentes tipologías contractuales como contrato de obra, contrato de consultoría, contrato de prestación de servicios, encargos fiduciarios y fiducia pública, y el que nos atañe: el contrato de concesión. En Colombia, se empieza a regular el contrato de concesión por el Decreto 222/1983, de 2 de febrero. Pero más adelante se incluye este tema más específico en la Ley 80/1993, de 28 de octubre, por la necesidad de que el Estado colombiano pretende regular toda la contratación estatal del país (González, 2016).

Sin embargo, se hace indispensable la inversión de capital privado para satisfacer los fines del Estado, y es donde el contrato de concesión de obra aparece, para incentivar la inversión de capital de los privados a los proyectos de infraestructura públicas. Cabe anotar que no se da de la manera más eficaz aquella inversión del sector privado y la Administración asume gran parte de los gastos para la ejecución de las obras. Para entender mejor el contrato de concesión de obra, encontramos un Estado que se denomina "concedente" y la otra parte "concesionario", que se encargará de la prestación, operación y explotación de un servicio público o la construcción de una obra, con la destinación al servicio o uso público. Cabe recalcar que el concesionario a cambio recibirá una remuneración o la explotación del bien o según las modalidades de pago que acuerden las partes (Ley 80/1993, de 28 de octubre, art. 32, núm. 4).

En la doctrina, encontramos que "el contrato de concesión de obra pública tiene por objeto la construcción, conservación o explotación de obras públicas nuevas, o la explotación, administración, reparación, ampliación de una obra pública antigua" (Caicedo, 2010, p. 43).

En efecto, la Corte Constitucional emite un concepto sobre el contrato de concesión, en el que nos explica que el Estado posee la obligación de prestar un servicio público, que, en algunas ocasiones, termina transmitiendo esa obligación a un particular, el cual se hace cargo de ella de una forma parcial o total según convengan las partes (Sentencia C-068/2009, de 10 de febrero).

Sin embargo, en los contratos de concesión de obra, el Estado trata de cumplir con los fines, los cuales le fueron encomendados y consagrados en la Constitución Política de Colombia, cuyo propósito es la prestación de los servicios públicos a través de diferentes tipos de infraestructuras públicas, velando por aquel interés general (Constitución Política, art. 365). No se han podido cumplir de la mejor manera, ya que en los contratos de concesión de obra, al Estado, en algunas ocasiones, le queda imposible materializar aquellas obras por el alto costo que conlleva 
realizarlas, y en las cuales necesita la intervención y financiación de terceros para llevarlas a cabo (Pardo, 2014).

Por lo descrito, aparecen las APP, las cuales cumplen una función importante que es inyectar capital privado, o bien de una persona natural, o bien de una persona jurídica, al diseño, construcción, operación o explotación de obras públicas o de servicios que el Estado quiere brindar a un colectivo general (Ley 1508/2012, de 10 de enero, art. 1).

Cabe resaltar que las APP son una nueva figura que nace para financiar, construir, operar y explotar las obras públicas del Estado, que se diferencian de los contratos de concesión de obra simple en la forma en que se desarrolla el proyecto y su financiación de la obra por el sector privado. Además que su gran enfoque es para la infraestructura vial del país sin olvidar otros sectores como salud, educación, etc. En la actualidad, para ejemplificar qué obra de gran magnitud se está llevando en Colombia, encontramos el contrato de concesión de obra por vía de las APP, en que el proyecto que se está ejecutando en la actualidad es la ruta del sol, la cual va a conectar el centro del país con la Costa Caribe. Por eso, la gran importancia de las APP para ejecutar grandes obras de infraestructura en el país, que sin ellas de por medio sería casi ineficaz que lo asumiera el propio Estado (Quiroz, 2014).

Sin embargo, debemos tener claro que los orígenes de las APP se ha dado en los Estados Unidos y Canadá. Al respecto, Urueta señala:

El fenómeno de la asociación público-privada se ha estudiado en varios países bajo diferentes terminologías. En ese sentido, en Estados Unidos, Canadá, Australia y Asia se lo ha llamado Partenariado Público Privado (PPP) y ha sido definido como una colaboración entre los agentes económicos, las organizaciones no gubernativas sin ánimo de lucro y las administraciones públicas, para la puesta en práctica de proyectos que son beneficiosos para los partners en particular y, de forma más general, para el conjunto de la colectividad. (2010, p. 30).

De igual forma, en la Unión Europea (UE), adoptan las APP con el término colaboración público-privada (CPP), y con el Sistema Europeo de Cuentas de 1995 (SEC 95), en el que se evidencia el sistema contable de un país, nos brindan una definición de las $\mathrm{CPP}$. Al respecto, señala Martínez:

Los acuerdos en los que una administración pública encarga a una sociedad, normalmente después de un procedimiento competitivo de concurso público, la 
construcción, financiación y explotación de un activo a lo largo de la duración del contrato. La sociedad privada será la encargada de cobrar directamente a los usuarios finales, que no son administraciones públicas, por la utilización efectiva de los servicios vinculados al activo. En este tipo de acuerdos, la mayor parte de los ingresos de la sociedad privada proceden de la prestación directa de los servicios. Bajo estas condiciones, los bienes de activo se registran en el balance de la sociedad privada, sin efecto sobre el déficit público y la deuda pública. (2006, p. 189)

Ahora bien, ya teniendo en cuenta dónde se originan las APP y la gran importancia para que el Estado colombiano haga un uso práctico de estas, debemos conocer nuestro marco normativo que las regula, de modo que la ley que da origen a las APP es la Ley 1508/2012, de 10 de enero, y, por consiguiente, otras normas que han regulado las APP en nuestro Estado hasta nuestra actualidad.

Encontramos el Decreto 1467/2012, de 6 de julio que reglamenta la estructuración y ejecución de los proyectos de las APP tanto de iniciativa pública como privada. Debemos tener en cuenta que cuando es de iniciativa pública la Administración debe aportar dinero a la realización de la obra y si es de iniciativa privada el inversionista hará el aporte total para la ejecución de la obra pública. Este decreto tiene en cuenta para la estructuración y ejecución del proyecto los siguientes indicadores de gestión: estándar de calidad, nivel de servicio, específico, medibles, oportunos, pertinentes, viables, unidad funcional de infraestructura que consiste en la ejecución que podría haberse realizado y contratado en forma independiente y autónoma, y que la unidad que se va a remunerar esté disponible y cumpla con niveles de servicio y estándares de calidad previstos (Decreto 1082/2015, de 26 de mayo, art. 2.2.2.1.2.2). Y finalmente los fondos públicos (Decreto 1467/2012, de 6 de julio, art. 2). Todo con la finalidad de que se lleve en los mejores ítems de calidad y servicio la obra por ejecutar (Cortés, 2013).

De igual forma, aparece la Resolución 3656/2012, de 20 de diciembre (art. 1), en la cual la Administración debe justificar si el medio idóneo son las APP para ejecutar la infraestructura, y para eso debemos tener un concepto favorable del Departamento Nacional de Planeación (DNP) o de la entidad de planeación de la respectiva entidad territorial (Resolución 3656/2012, de 20 de diciembre, art. 1). Lo vemos reflejado en la Ley 1753/2015, de 9 de junio, por la cual se expide el Plan Nacional de Desarrollo 2014-2018 “Todos por un nuevo país”, para lo cual debemos tener en cuenta lo descrito en la Ley 1753/2015, de 9 de junio (art. 206). 
Sin embargo, cabe recalcar, el DNP en 2016 describe qué requisitos deben reunir las entidades públicas para recibir un concepto favorable y recomendaciones cuando aquellas desarrollen proyectos por medio de las APP, teniendo en cuenta el plan sectorial y la necesidad de implementar o ejecutar la infraestructura en aquel territorio (Resolución 1464/2016, de 1 de abril, art. 1).

Finalmente, la última normativa que regula algunos aspectos frente a las APP en la actualidad es la Ley 1882/2018, de 15 de enero, y uno de los aspectos por precisar es que la selección de las APP se hará bajo la modalidad de licitación pública, por la cual se pretende que haya transparencia y un equilibrio económico entre las partes (art. 13, par. 4).

Es interesante recalcar que las APP no hacen parte de las tipologías contractuales en la actualidad, además son un modelo diferente del modelo tradicional de contratación en el Estado, sin olvidar que la Administración puede ahorrar gran parte del gasto público de esas obras por medio de estas figuras de las APP, para invertir en otras políticas públicas a cambio de una retribución para el contratista del sector privado, ya sea por medio de tasas, tarifas, porcentajes, etc.

Como se había mencionado, las "APP permiten al gobierno evitar o diferir el gasto en infraestructura sin renunciar a sus beneficios. Esta puede ser una ventaja especialmente atractiva para los gobiernos cuya capacidad actual de gasto se encuentra restringida pero que están relativamente libres de restricciones para comprometer gastos futuros" (Akitoby, Hemming y Schwartz, 2007, pp. 10-11).

Cabe reseñar que las APP, cuando han ejecutado las obras públicas y están concesionadas para las vías, deben "conservar en buen estado el corredor vial y no permitir su avanzado deterioro por carencia de recursos del presupuesto nacional" (Contraloría General de la Nación, 2001, p. 9).

Por esto, es importante resaltar que los contratos de concesión de obra pública por vía de las APP benefician a un colectivo general, en que la relación entre la Administración y el contratista del sector privado favorece a las dos partes y, sobre todo, el avance económico, social y de infraestructura en el país, que tanto lo necesita. Gracias a esta figura, el dinero que el Estado no aporta en la inversión de la infraestructura se puede ver reflejado en otras políticas públicas que beneficien a todos los colombianos. 


\section{Fortalezas del contrato de concesión de obra en las APP}

Es importante determinar las fortalezas que nos brinda la utilización de las APP en el país, para el desarrollo de la infraestructura y prestación del servicio público en que el Estado es el garante, como lo consagra el artículo 365 de la Constitución Política de 1991. Por consiguiente, se enumeran las fortalezas más representativas de esta figura, utilizada con mayor frecuencia en la contratación estatal en la actualidad.

\section{Se le paga al contratista en la medida en que preste un servicio de calidad y eficiente en la infraestructura}

Si bien es cierto que el contratista debe propender por una calidad alta en la obra ejecutada, como en su diseño y materiales de construcción utilizados, en que prime la eficacia y la eficiencia en la entrega de la obra, y en que la Administración debe darse cuenta de la disponibilidad de ella misma, que pueda ser utilizada al $100 \%$ por la comunidad, si es así, en retribución el contratista recibirá una contraprestación económica según lo acordado entre las partes. De acuerdo con Moore:

Centrarse, en cambio, en lo que realmente produce el trato y cómo los costos y los beneficios de la producción conjunta son distribuidos. Para evaluar en qué medida un trato es eficiente, justo y legítimo, debemos mirar hacia la estructura del trato tal cual fue hecho y en su rendimiento al ser ejecutado. (2006, p. 11)

Por tal motivo, estos tres factores de disponibilidad, calidad y retribución deben satisfacer la voluntad del Estado, siempre y cuando el privado lo cumpla con eficiencia, planeación, y a cambio reciba una retribución por la obra. Al respecto, Hernández indica:

Si bien la disposición de la infraestructura es muy importante y se materializa, entre otros aspectos, en regular el acceso y la utilización de la misma por parte de quienes deseen hacerlo, el establecimiento del derecho de explotación sobre la frecuentación de la obra por los usuarios y no tanto sobre su puesta a disposición, viene a significar el derecho de explotación en sí mismo, como derecho del privado y elemento propio de este tipo de acuerdo. (2014, p. 28) 
En Colombia, el DNP (2018b) reitera lo importante de la calidad del servicio de la infraestructura, o de lo contrario no se le pagará al privado, argumentando lo siguiente:

No se paga si el servicio no es bueno: Las APP facilitan la provisión y el mantenimiento de infraestructura, dentro de unos parámetros de disponibilidad y calidad. No se invierte en proyectos inmaduros: Adecuada estructuración de proyectos en cuanto a estudios, análisis de riesgos, evaluación socioeconómica y justificación de la modalidad de contratación. Cumplimiento de plazo de construcción: No hay anticipos, el pago se realiza de acuerdo con el cumplimiento y disponibilidad del servicio. Precios realistas: Se limitan las adiciones en recursos al 20 \% del valor del contrato.

De lo anterior se puede deducir que al privado en un contrato de concesión de obra por vía de las APP se le pagará en la medida en que tenga montada toda la infraestructura a nivel de construcción, operación, mantenimiento y, por ende, en funcionamiento, teniendo en cuenta varios parámetros, como disponibilidad en el servicio, calidad y eficiencia, de lo contrario, no se le efectuará el pago al contratista.

En Sentencia C-595/2014, de 20 de agosto, se reitera que "bajo el nuevo enfoque de asociaciones público-privadas se busca: (1) estructurar los mecanismos de pago del Gobierno y evaluar la infraestructura en función del servicio que se ofrece a los beneficiarios, medido en términos de desempeño, calidad y disponibilidad”.

Finalmente, hemos observado lo importante en cuanto a la disponibilidad del servicio y su calidad en la obra, como lo confirma Pardo, "en otras palabras lo que garantiza la existencia de la externalidad durante la fase de implementación del proyecto, es la definición precisa de las condiciones de calidad esperadas por el Estado" (2013, p. 33).

\section{Reducción del endeudamiento y déficit público del Estado}

Es preciso señalar que las APP han servido para que el Estado no posea un endeudamiento y déficit público alto, por la construcción de alguna infraestructura que necesite ejecutar, dado que las APP son un tipo de figura que busca que el sector privado realice la mayor parte de la inversión. Por consiguiente, el Estado no tendría la carga de asumir aquellos gastos, por los que le sería imposible llevar a cabo las obras públicas para el beneficio de la colectividad en general (Rodríguez, 2016). 
Por lo anterior, el Estado, si asumiera los gastos de aquellas infraestructuras tan elevadas en costo, descuidaría la inversión en otros campos de acción, porque su endeudamiento y gasto público se elevaría por realizar las obras públicas. Al respecto, Vives señala:

Los gobiernos se han visto forzados a fijar su atención en resolver la crisis y mitigar sus impactos y se han concentrado en temas relativos al sistema financiero, de gobernanza privada y de inversión pública, descuidando las demás responsabilidades ambientales y sociales. De hecho, en algunos países el gobierno ha tenido que reducir la intensidad de su acción social ante los necesarios ajustes fiscales. (2010, p. 17)

Por eso, es tan importante la intervención de las APP, porque la Administración no posee el músculo financiero y la experticia de los privados para ejecutar las obras encomendadas, en relación con los fines del Estado en la contratación estatal. Por ende, el gasto público se reduce porque "el Estado no tiene recursos suficientes para todo [...] Le faltan manos, tiempo, dinero y, en muchos casos, conocimientos y experiencia. Por donde miremos falta algo por construir o mejorar, un servicio público básico que prestar" (Heros, 2009, p. 388).

\section{Las APP como impulsadoras en el crecimiento y desarrollo de la infraestructura del país}

Si bien es cierto que el país ha utilizado los mecanismos tradicionales de contratación estatal, y aun así tenemos un déficit en infraestructura que limita un desarrollo económico y social en nuestro territorio, debido a que no tenemos, por ejemplo, vías de infraestructura competitivas, y en otros sectores en los que la infraestructura debe desarrollarse de igual forma con agilidad y eficacia, como en salud, educación, deportes, etc., cuando aparece la figura de las APP en el país, no solo se encuentra un déficit en infraestructura por la incapacidad del Estado en poder ejecutarlas, por eso el papel importante de las APP en el país para desarrollarlas, de modo que lo primero que se debe hacer es atraer inversionistas privados. Según Serrano,

atraer inversionistas privados a la ejecución y financiamiento de proyectos de infraestructura en transporte ha sido exitosa en otras latitudes. En Colombia se ha hecho un gran esfuerzo para su promoción y utilización; le permite al Estado 
posponer el uso de recursos públicos, así el pago se haga a través de vigencias futuras. (2010, p. 115)

Además de "atraer inversionistas de largo plazo con suficiente capacidad financiera que no solo construyan la infraestructura sino que la operen y la mantengan" (DNP, 2018b). Por eso, el sector privado desempeña un papel importante, que es aumentar la eficacia en la ejecución de la obra, ya que es "la acumulación de las distintas fases de un proyecto en manos del mismo sujeto privado quien consigue explotar sinergias entre ellas, incorporando diseños más innovadores y a un coste más efectivo" (Fuertes, 2007, p. 239).

El desarrollo de infraestructura del país va aumentar el desarrollo económico y social, por ejemplo, la construcción de la ruta del sol que comunicará el centro del país con la Costa Caribe o la primera APP en el sector de cultura en la remodelación del coliseo El Campín cuya "inversión privada de $\$ 70$ mil millones y un nuevo escenario consolidado con un área de 37 mil metros cuadrados para convertirlo en uno de los más modernos de Latinoamérica" (HSBnoticias.com, 2015).

Además, los importantes avances en "la confianza que el Estado deposita en los empresarios del sector privado para la realización de infraestructuras fundamentales e, incluso, estratégicas para el desarrollo económico y social del país” (Loo, 2015, p. 332).

\section{Se identifican los riesgos para reducir los retrasos, los costos suplementarios y las renegociaciones del proyecto}

En la relación entre el privado y el Estado en el momento de celebrar un contrato de concesión de obra por vía de las APP, se deben identificar los riesgos previsibles, fortuitos, analizar el marco político, económico y social del país, la necesidad de realizar la obra. Por ello, es de gran importancia la planeación para la identificación de los riesgos, además se debe asignar la parte que va asumir a la totalidad de los riesgos, cómo han de mitigarse y manejarlos de la mejor forma: para este caso, recae toda la responsabilidad en el privado en la realización de la obra.

La finalidad de la identificación de los riesgos es tratar de que se dé la "reducción o eliminación de retrasos, costos suplementarios y renegociaciones" durante la ejecución del contrato (Mejía, 2018, p. 9). Al respecto, Alborta, Stevenson y Triana señalan que 
la identificación, cuantificación, asignación y mitigación de riesgos, [...] constituyen el mecanismo central de un esquema de APP. Independientemente de la naturaleza de los contratos de APP y de la base de su retribución, la asignación de riesgos y responsabilidades en los acuerdos de APP debe responder a principios realistas de equidad y balance para ambas partes. Si las evaluaciones de riesgo no son realistas, o se asignan demasiados riesgos al sector privado, los inversionistas privados no se interesan o cargan un costo adicional por el riesgo. (2011, p. 65)

Es de recalcar que no solo puede existir inversión privada de los nacionales, sino también de los extranjeros, por ende, se debe proteger la inversión de las multinacionales extranjeras. El Estado deber tener claros y expresos aquellos riesgos, los cuales va a asumir el sector privado para que los extranjeros no tengan dudas frente a la inversión que van a realizar en el país. Al respecto, Wills indica que,

para contrarrestar el bajo nivel de capacidad de financiación en infraestructura, los países en vías de desarrollo han acudido cada vez más a la inversión del sector privado. Esta inversión es una buena opción para los Estados, pues no solo ofrece una manera de financiar los proyectos en infraestructura, sino que también transfiere los riesgos financieros de los proyectos al sector privado. (Wills, 2011, p. 22)

Debemos dejar claro que en teoría el que asume los riesgos es el concesionario, ya que en varios pronunciamientos de la doctrina se reitera esta afirmación: "Todas aquellas actividades necesarias para la adecuada prestación o funcionamiento de la obra o servicio por cuenta y riesgo del concesionario y bajo la vigilancia y control de la entidad concedente" (Millán, Blanco y Guecha, 2017, p. 206). Pero, cuando se presentan renegociaciones de los contratos, el Estado debe compartir algunos riesgos con el privado, "sin embargo en la práctica, los contratos contienen ambigüedades y a menudo el gobierno debe compartir los riesgos durante la vigencia de los mismos, debido a que los contratos se renegocian" (Engel, Fischer y Galotovic, 2014).

Por último, es interesante que el Estado tenga en cuenta la cláusula de renegociación, porque puede afectar de cierta forma el interés del privado por participar en las concesiones de obra por vía de las APP, como lo reitera Leudo y Blanco (2016): "Si bien la renegociación de contratos de concesión puede ser objeto de críticas, ya que una de sus consecuencias es que se afecta el éxito y la credibilidad de la vinculación del capital privado a la generación de infraestructura” (p. 175). 


\section{Deficiencias del contrato de concesión de obra en las APP}

Se demuestra con la expedición de la Ley 1508/2012, de 10 de enero y el Decreto 1467/2012, de 6 de julio que, a pesar de que exista una regulación en cuanto a los contratos de concesión de obra por vía de las APP en el país, esta figura jurídica presenta unas deficiencias las cuales abordaremos a continuación.

\section{Solo se está aplicando en mayor medida en los contratos de concesión de obra}

Una de las razones del porqué se están aplicando con mayor fuerza los contratos de concesión de obra en los de cuarta generación es en definitiva buscar el crecimiento económico y aumentar la competitividad del país a través del desarrollo de infraestructura y transporte. Han existido de primera, segunda y tercera generación, todos con avances y deficiencias, en el desarrollo de infraestructura vial en todos nuestros departamentos; para ello, se va mejorando y adecuando todo el sistema normativo legal en cuanto a contratación estatal. Para este caso, las concesiones de cuarta generación puedan cubrir aquellas deficiencias del pasado en las otras concesiones, y por eso se expide la Ley 1508/2012, de 10 de enero, para tratar de satisfacer aquellas necesidades en cuanto a la malla vial en Colombia (Rodríguez, 2014).

Las concesiones de cuarta generación lo que buscan es "la construcción y la rehabilitación de más de 7.000 kilómetros de vías, que contemplan dobles calzadas (más de $1.370 \mathrm{~km}) ; 1.300$ viaductos $(146 \mathrm{~km})$ y 141 túneles $(125 \mathrm{~km})$ ” (MBD, Ingeniería de Valor S. A., 2016). Pero existe un antecedente, en el que el DNP empieza a visualizar que el Estado debe ser más competitivo en infraestructura vial. Solarte señala:

En el año 2010, el DNP financió el Plan Maestro de Transporte 2010-2032, con el objetivo de identificar los principales lineamientos para el desarrollo sectorial, concentrándose en la necesidad de incrementar la oferta de infraestructura bajo criterios técnicos de priorización, para lo cual se establecieron cinco grandes componentes: i) Estructuración Institucional, ii) regulación y servicios de transporte, 
iii) oferta de infraestructura, iv) financiamiento del sector de transporte del PMT y

v) desarrollo logístico. (2013, p. 47)

Por lo anterior, es claro que el Estado colombiano le apuesta al desarrollo sectorial de infraestructura del país, pero aparece una deficiencia en cuanto a la forma en que se utilizan las APP: que esta figura solo se está aplicando en mayor medida en los contratos de concesión de obra por vía de las APP para las concesiones de cuarta generación. Cabe recordar que en Colombia se están manejando ocho proyectos de infraestructura de este calibre. Esto nos indica que hay mayor interés del Estado colombiano en apostarle a su plan nacional de desarrollo en cuanto a la modernización de infraestructura vial de todo nuestro territorio. Es importante recalcar que las APP aplican para cualquier tipo de contrato; las APP no es un tipo contractual más, es una forma de pago al contratista, dependiendo de los factores de disponibilidad de servicio y calidad de la obra.

\section{Requiere una suma fuerte de presupuesto por parte del sector privado}

Los contratos de concesión de obra requieren una suma fuerte de inversión en recursos y logística. Además, existe una limitante del porqué otros contratos de concesión de obra no van por vía de las APP. El monto establecido para poder acceder por este medio, que se encuentra estipulado en el artículo 3, parágrafo 1, de la Ley 1508/2012, de 10 de enero, en la que el monto mínimo es de 6000 salarios mínimos mensuales legales vigentes (SMMLV) para optar por la vía de la APP. Esto quiere decir que, si el SMMLV para 2018 es de COP 781 242, para acceder a un contrato por medio de una APP, el valor sería de COP 4687 452. 000. Al respecto, Baptiste señala:

Solo proyectos muy grandes pueden realizarse con actores privados que cuenten con esa gran cantidad de dinero, impidiendo que actores privados que tengan propuestas de proyectos más simples y que no alcancen a ese monto o no tengan esa capacidad monetaria no puedan presentar proyectos de cooperación público-privada, pudiendo dejar de lado ciertas oportunidades que tiene el Estado de poner en marcha proyectos no tan grandes pero que bajo un esquema de asociación públicoprivada puede facilitar su realización. (2013, p. 26) 
Una de las pocas entidades del Estado que está ejecutando los contratos de concesión de obra por vía de las APP es la Agencia Nacional de Infraestructura

Por medio del Decreto 4165/2011, de 3 de noviembre, se crea la Agencia Nacional de Infraestructura (ANI), cuya finalidad es el desarrollo óptimo vial de todas las carreteras del país, además de la importancia a primera vista de la vinculación de capital privado por medio de APP a los contratos de concesión de obra. Por tal razón, el Gobierno de turno modifica la naturaleza jurídica del Instituto Nacional de Concesiones (INCO), para dar paso a la nueva ANI y con ello ser más eficiente y en lo posible reducir los altos niveles de incumplimientos, anticipos, desfalcos, corrupción, servicio y calidad de las obras entregadas al Estado (art. 1).

Pero surge una gran inquietud del porqué la ANI es la única entidad del Estado que maneja las concesiones de infraestructura vial en el país, habiendo otras entidades como el Instituto Nacional de Vías (Invías), creado por el Decreto 2171/1992, de 30 de diciembre, por el cual se reestructura el Ministerio de Obras Públicas y Transporte como Ministerio de Transporte y se suprimen, fusionan y reestructuran entidades de la rama ejecutiva del orden nacional.

Teniendo en cuenta lo anterior, Invías tiene como objeto los proyectos relacionados con la infraestructura vial a cargo del Estado, pero existe una limitante frente a esos proyectos, cuyo objeto es que se hará cargo de las concesiones viales, excepto de las concesiones de infraestructura de cuarta generación en el país. En consecuencia, la única entidad competente para manejar los temas de concesiones de obra por vía de las APP es la ANI; pero, como lo explicamos por el problema que hubo en la construcción de la ruta del sol, fase 2 , se le adjudicó cierta competencia al Invías en esta contratación, lo cual abre una luz en el futuro para que estas dos entidades o las demás afines puedan complementarse con la ANI, para el desarrollo de las vías de cuarta generación en el país.

\section{Falta de capacitación en las entidades estatales}

Aunque lleva poco tiempo la Ley 1508/2012, de 10 de enero, las entidades del Estado que manejan contratos por vía de las APP deben preocuparse por la capacitación de sus funcionarios en cuanto a lo concerniente al procedimiento de esta figura, los beneficios que puede traer si se adjudica este contrato, etc. Han de tener claro que si es de iniciativa del Estado el proyecto, qué sucede, si es de iniciativa 
privada, cuáles son los presupuestos que debe asumir el contratista, cuánto es el monto de la cuantía por la cual se puede acceder a una APP, todo su marco legal, etc. Cabe recordar que no solo la ANI maneja contratos por APP, sino que otros sectores, como la Secretaría de Educación y el sector de la salud, deben preocuparse por la formación de sus empleados para obtener un conocimiento idóneo de cómo funcionan las APP.

Es importante capacitar a la entidades del Estado, por ello el Ministerio de Hacienda y Crédito Público (MinHacienda) las está fomentando: "Se desarrolla una serie de capacitaciones que tienen como finalidad orientar y dar lineamientos a las entidades territoriales sobre el funcionamiento y las ventajas de la utilización de los proyectos de APP's para el desarrollo social y económico de las diferentes regiones del país" (s. f., p. 4).

\section{Demora en el trámite de licencias ambientales}

Las licencias ambientales en el país están a cargo de la Autoridad Nacional de Licencias Ambientales (ANLA) por el Decreto 2820/2010, de 5 de agosto:

Artículo 3. ${ }^{\circ}$ Concepto y alcance de la licencia ambiental. La Licencia Ambiental, es la autorización que otorga la autoridad ambiental competente para la ejecución de un proyecto, obra o actividad, que de acuerdo con la ley y los reglamentos pueda producir deterioro grave a los recursos naturales renovables o al medio ambiente o introducir modificaciones considerables o notorios al paisaje; la cual sujeta al beneficiario de esta, al cumplimiento de los requisitos, términos, condiciones y obligaciones que la misma establezca en relación con la prevención, mitigación, corrección, compensación y manejo de los efectos ambientales del proyecto, obra o actividad autorizada.

La licencia ambiental llevará implícitos todos los permisos, autorizaciones o concesiones para el uso, aprovechamiento o afectación de los recursos naturales renovables, que sean necesarios por el tiempo de vida útil del proyecto, obra o actividad (Decreto 2820/2010, de 5 de agosto, art. 3).

En ocasiones, la demora en el trámite o procedimiento para solicitar la licencia ambiental para un proyecto de infraestructura vial puede demorarse bastante tiempo teniendo en cuenta aspectos para conceder la licencia ambiental como 
medio ambiente, transformación en el paisaje o afectación en algún hábitat. Por consiguiente, las demoras en este trámite pueden provocar algunos retrasos en la iniciación de la obra.

\section{Propuestas}

En esta sección, abordaremos unas propuestas en cuanto a las concesiones de obra cuando se gestionan por vía de las APP en el país, las cuales se describen a continuación.

\section{Aplicación de las APP}

El uso de las APP en gran medida se están aplicando en los contratos de concesión de obra; para este caso, las vías de cuarta generación, lo importante es que se empiecen a aplicar las APP, por ejemplo, en los contratos de obra y en los contratos de prestación de servicios, para que haya un desarrollo más rápido, ágil en lo productivo y económico del país, teniendo en cuenta que las APP reducen el costo para el Estado, pero a cambio recibe una infraestructura en ítems de construcción, operación y mantenimiento, bajo los estándares de calidad y disponibilidad en el servicio. Por ende, debería aplicarse con más frecuencia en otros tipos de contrato.

\section{Regular el monto para acceder a la figura de las APP}

En la actualidad, el monto establecido para poder acceder por este medio se encuentra estipulado en el artículo 3, parágrafo 1, de la Ley 1508/2012, de 10 de enero, en la que el monto mínimo es de 6000 SMMLV para optar por la vía de las APP. Esto quiere decir que, si el mínimo para 2018 es de COP 781 242, para acceder a un contrato por medio de una APP, el valor sería de COP 4687452 000. Por ende, los proyectos de mediana y pequeña escala no tendrían cabida, lo que hace necesario una modificación en el artículo 3 de la Ley 1508/2012, de 10 de enero, para que disminuyera el monto y así promover las iniciativas de proyectos medianos y pequeños de infraestructura, y de cierta forma contribuir al desarrollo económico y productivo del país, de una forma más ágil y eficaz. 


\section{Que la Agencia Nacional de Infraestructura no fuera la única que manejara los contratos de concesión de cuarta generación}

En el país, la entidad encargada de manejar las concesiones de cuarta generación es la ANI; sin embargo, por los problemas de la concesión de la ruta del sol, fase 2, al Invías se le asignó una función en cuanto a esa concesión, con lo que se ha visto algún adelanto así sea pequeño en lo referente a que la ANI no sea el único ente que regule este tipo de concesiones, que se verá en el Decreto 431/2018, de 5 de marzo, artículo 2: "Contracredítese del presupuesto de gastos de inversión de la vigencia fiscal 2018 de la Agencia Nacional de Infraestructura (ANI), la suma de doscientos cincuenta mil millones de pesos (\$250.000.000.000.00) moneda corriente".

Pero a la ANI se le recortó el presupuesto de 2017, de COP 400000000000 a 2018, a COP 250000000 000; esos COP 150000000000 que se le redujeron se los asignaron al Invías (Decreto 431/2018, de 5 de marzo, art. 3). Dan para muchas interpretaciones estas asignaciones de recursos, pero lo más importante es que existiera una regulación legal para que el Invías pudiera ser competente frente a las concesiones de cuarta generación en el país, para que asimismo no se depositara toda la responsabilidad en la ANI, sino que se distribuyeran funciones frente a ese tipo de concesiones. Claro ejemplo el que pasó en el proceso de adjudicación de licitación del nuevo contratista para el proyecto de la ruta del sol, fase 2.

\section{Capacitación en las entidades estatales}

La Ley 1508/2012, de 10 de enero, en primera instancia, se consagró con la finalidad de utilizar las APP en los proyectos de infraestructura vial, transporte y movilidad. Aunque su aplicación es detallada y procedimental, no es restrictiva sino amplia. Por ende, debería trabajarse en la capacitación e información en todas las entidades del Estado de cómo funcionan las APP, sus beneficios, el conocimiento en su procedimiento, así como que estas entidades puedan iniciar proyectos por medio de las APP en otros sectores diferentes del de transporte y de la malla vial, entendiendo que las APP lo que buscan es el aumento en sectores productivos del país, como en lo económico y social. Como lo reitera Hernández, "su aplicación se desarrollará de acuerdo con la coyuntura jurídica, económica y política y las necesidades de infraestructura de bienes y servicios que la administración considere se deban proveer a través de Contratos de Asociación Público Privada” (2016, p. 104). 
Además, "con la expedición de la Ley 1508, apareció en el panorama de la contratación estatal colombiana la posibilidad de estructurar ya sea de Iniciativa Pública o Privada, proyectos bajo el marco de las APPs, y que tiene toda la potencialidad de generar proyectos de carácter social, entre otros" (Huertas, 2017, p. 43).

\section{Licencias ambientales}

Para un desarrollo eficaz y ágil por parte de la Administración en cuanto al desarrollo de infraestructura en el país, o cuando la iniciativa es del sector privado, sería recomendable la disminución en los tiempos de trámite, simplificación de la documentación en la adquisición de las licencias ambientales o en la adquisición de los predios para el desarrollo de infraestructura vial, transporte o movilidad. Con ello habría que realizar una modificación en el marco legal de las normas que regulan estas situaciones, con el fin de un desarrollo rápido en la infraestructura tendiente a la disminución de tiempo para empezar a ejecutar la obra.

Por consiguiente, es "necesario entonces adoptar medidas para evitar que la concreción de estos riesgos impida la ejecución de las obras que necesita el país para salir del atraso en el que se encuentra" (Araujo y Sierra, 2013, p. 189).

\section{Etapa de prefactibilidad}

Cuando la iniciativa es del sector privado para un proyecto de infraestructura pública, aquel debe asumir el total de los costos de la estructuración de la propuesta en la etapa de prefactibilidad, por su propia cuenta y riesgo. Pero, aparte de asumir todos los costos, ha de esperar que la entidad a la que fue presentada la estructuración del proyecto decida si le interesa, y por ende tiene la competencia de decidir si opta o no por la propuesta que le fue presentada. "Para proyectos de APP de iniciativa pública, la entidad contratante asume todos los gastos de prefactibilidad - estudios, evaluación de costos, justificación, etc.- requeridos para abrir el proceso de selección de contratistas" (Poveda, 2015, pp. 35-36).

Por lo anterior, habría más iniciativas por los privados para la presentación de proyectos de infraestructura, siempre y cuando el Estado asumiera la totalidad o la división de costos con los particulares en la estructuración de la propuesta, para el bienestar e interés general de toda la comunidad. 


\title{
Continuidad en las políticas públicas
}

En cuanto a la implementación de políticas públicas, señala Córdoba:

\begin{abstract}
La implementación de las políticas públicas se observó inicialmente, como un proceso de interacción entre el establecimiento de metas y las acciones generadas para lograrlas, luego vista desde la acción para la corrección de los defectos y obstáculos, ocasionados por la interacción entre varios actores, con intereses, responsabilidades, operaciones y decisiones diversas y por último, vista desde la identificación de fallas derivadas de las limitadas capacidades de los Gobiernos y los problemas en las estructuras institucionales. (2017, p. 47)
\end{abstract}

De lo anterior es importante reforzar estructuralmente las entidades del Estado, que tengan claro sus metas a corto, mediano y largo plazo, y que vaya en concordancia con las políticas públicas nacionales, que trabajen conjuntamente por el bien de la comunidad.

Es importante recalcar que las concesiones de obra por vía de las APP pueden ser hasta de treinta años; por consiguiente, existen "los problemas asociados a los contratos a largo plazo" (Mendoza, 2012, p. 57). Así que es importante la continuidad de políticas públicas a ese tipo de concesiones de largo plazo. En nuestro Estado, los gobernantes se posesionan cada cierto tiempo y lo más importante es que haya una sostenibilidad en cuanto a los proyectos que se están realizando, sin importar el gobernante de turno, continuando con las políticas públicas, y así procurar un bienestar social de toda la población, que se beneficiaría con la culminación exitosa de cualquier proyecto de infraestructura que promueva el aumento económico y social en nuestro territorio nacional.

También es necesario que las entidades territoriales se asocien para que trabajen por ese fin en común en la continuidad de las políticas públicas. Como lo afirma Hernández,

el principio del desarrollo de la descentralización y la asociatividad, tal como lo señala Sepúlveda, se puede desarrollar mucho mejor con la tipología institucional; ya que fomenta la formación de una persona jurídica entre los dos sectores, proyectándose a lo largo del tiempo y fortaleciendo la sostenibilidad de los proyectos que ejecuta; y es más conveniente porque da mayor respaldo y garantías a las entidades que se decidan asociar. (2016, p. 40) 
Cabe recalcar que, cuando existen políticas públicas estables en el tiempo, "los actores privados $[\ldots]$ se involucran en este tipo de asociaciones, donde a su vez adquieren responsabilidades y obligaciones porque tienen claro su viabilidad a nivel económico y la sostenibilidad de las alianzas en el tiempo" (Olave, 2016, p. 24).

Además, para que se fortalezcan y continúen aquellas políticas públicas, se deben manejar de una forma adecuada y responsable por parte de los gobernantes al proponer la vigencia fiscal y las vigencias futuras a que haya lugar, sin afectar los recursos destinados al desarrollo de infraestructura vial en el país. La vigencia fiscal hace referencia a que el "año fiscal comienza el $1^{\circ}$ de enero y termina el 31 de diciembre de cada año. Después del 31 de diciembre no pueden asumirse compromisos con cargo a las apropiaciones del año fiscal" (Ministerio del Interior, 2013). En cuanto a las vigencias futuras, estas aluden a la autorización para asumir obligaciones que afecten el presupuesto de vigencias siguientes, que pueden ser ordinarias, cuya ejecución se inicia afectando el presupuesto de la vigencia en curso, y excepcionales, cuyas obligaciones afectan el presupuesto de vigencias fiscales futuras y no cuentan con apropiación en el presupuesto de la vigencia en que se concede la autorización y son aprobadas por el Consejo Superior de Política Fiscal (Confis) (ANI, s. f.).

\section{Comité de evaluación de las APP}

Cuando las ofertas son de iniciativa privada, es necesario fortalecer el comité de evaluación en la selección de personas idóneas y con un nivel académico alto en cada entidad estatal, y si no existiere crearlo, para que evalúe los proyectos de las APP en el país, y de ese modo evaluar de manera concienzuda los pros y los contras de aquel proyecto presentado por el sector privado para aceptar su favorabilidad o no. Como lo reitera Reina: "También [se] identifica como aspecto a mejorar la falta de un comité de evaluación de las ofertas de APP, ya que estos son necesarios dados [sic] la gran complejidad de los proyectos" (2015, p. 28).

Sin embargo, la entidad debe tener claro que en el momento de contratar con un sector privado el comité experto debería tener en cuenta, según Gómez y Ruiz,

que se haga un proceso de estructuración técnico, legal y financiero muy estricto, en el que se defina con claridad cuáles serán los parámetros, niveles de servicios y estándares de calidad, que permitirán entender que la obra se encuentra disponible y que el servicio se presta de acuerdo con las necesidades, para garantizar el éxito 
de la contratación y lograr la mejor forma de invertir los recursos públicos. (2015, p. 69)

\section{Fortalecer los sistemas de auditorías}

Es necesario tener un control más estricto frente al desarrollo de concesiones de obra por vía de las APP, aparte de los entes de control como la Contraloría General de la República, la Procuraduría General de la Nación, la Superintendencia de Sociedades, el DNP, la ANI, etc. Se debe llevar un control y vigilancia permanente y continuo en las obras de infraestructura que se están desarrollando, con el fin de que cumplan con los estándares de calidad, tiempo, eficacia y servicio. Por eso, se deben fortalecer los sistemas de auditorías periódicamente para lograr detectar alguna falla en la ejecución y el funcionamiento de la obra y tomar las acciones correspondientes en el momento indicado.

\section{Conclusiones}

Con esta investigación se logró llegar a unas conclusiones en cuanto a los contratos de concesión de obra gestionados por las APP. Se determinó todo el marco normativo, las fortalezas y las deficiencias que rodean el contrato de concesión de obra cuando se gestiona por vía de las APP. Además de presentar unas propuestas o recomendaciones a esta tipología contractual que nace a partir de la Ley 1508/2012, de 10 de enero, en la que se regula la contratación.

Uno de los grandes beneficios de las APP, cuando el Estado contrata con el sector privado, es que a este último, el Estado, le pagará en la medida en que tenga montado a toda la infraestructura a nivel de construcción, operación, mantenimiento $y$, por ende, en funcionamiento, teniendo en cuenta varios parámetros, como la disponibilidad en el servicio, la calidad y la eficiencia de esta; de lo contrario, no se le efectuará el pago al contratista.

Es importante la intervención de las APP, porque la Administración no posee el músculo financiero y la experticia del sector privado para ejecutar las obras encomendadas, en relación con los fines del Estado en la contratación estatal; por ende, el gasto público se reduce, y se puede invertir en otras necesidades básicas que necesita la población, por el ahorro que hace el Estado al acudir a las APP. 
Cuando aparece la figura de las APP, se encuentra un déficit en infraestructura por la incapacidad del Estado en poder ejecutarlas; por eso, el papel importante de las APP en Colombia para desarrollarlas, de modo que lo primero que se debe hacer es atraer inversionistas privados, y si es el caso inversionistas extranjeros, con el fin de seguir con el crecimiento económico y social del país, gracias al desarrollo ágil y eficaz de infraestructuras u obras por medio de esta figura jurídica.

Las APP nos brinda un gran avance en tanto se establecen los riesgos que debe asumir el sector privado durante la ejecución de la obra, lo cual genera mayor tranquilidad para el Estado colombiano, en el momento en el que el contratista inicie la ejecución de la infraestructura; por consiguiente, el Estado sale como gran beneficiario de esta alianza con el concesionario, porque toda la responsabilidad recae en el sector privado.

El uso de las APP en gran medida se están aplicando en los contratos de concesión de obra, para este caso las vías de cuarta generación. Lo importante es que se empiecen aplicar las APP, por ejemplo, en los contratos de obra y en los contratos de prestación de servicios, para que haya un desarrollo más rápido, ágil en lo productivo y económico del país, teniendo en cuenta que las APP reducen el costo para el Estado.

En la actualidad, el monto establecido para poder acceder a la figura de las APP se encuentra estipulado en el artículo 3, parágrafo 1, de la Ley 1508/2012, de 10 de enero, en la que el monto mínimo es de 6000 SMMLV para optar por la vía de las APP, lo cual quiere decir que las APP solo son para proyectos de gran escala o envergadura, por ende, para inversionistas que posean un gran poder adquisitivo que quieran licitar por medio de esta figura jurídica.

En el país, la entidad encargada de manejar las concesiones de cuarta generación es la ANI, sin embargo, por los problemas de la concesión de la ruta del sol, fase 2, al Invías se le asignó una función en cuanto a esa concesión, aunque se ha visto algún adelanto así sea pequeño, con respecto a que la ANI no sea el único ente que regule este tipo de concesiones.

Se debe trabajar en la capacitación e información en todas las entidades del Estado, de cómo funcionan las APP, sus beneficios, conocimiento en su procedimiento, $y$ de modo que estas entidades puedan iniciar proyectos por medio de las APP en otros sectores diferentes del de transporte y de la malla vial, entendiendo que las APP lo que buscan es el aumento en sectores productivos del país, como en lo económico y social. 
Se deben disminuir los tiempos de trámite, simplificación de la documentación en la adquisición de las licencias ambientales o en la adquisición de los predios para el desarrollo de infraestructura vial, transporte o movilidad en aras de la agilidad y eficacia de la ejecución de la infraestructura.

Habría más iniciativas del sector privado para la presentación de proyectos de infraestructura, siempre y cuando el Estado asumiera la totalidad o la división de costos con los particulares en la estructuración de la propuesta, en la etapa de prefactibilidad.

Es importante la continuidad de políticas públicas a ese tipo de concesiones a largo plazo. En nuestro Estado, los gobernantes se posesionan cada cierto periodo y lo más importante es que haya una sostenibilidad en cuanto a los proyectos que se están realizando, sin importar el gobernante de turno, continuando con las políticas públicas y así poder cumplir con éxito la ejecución de la infraestructura.

Cuando las ofertas son de iniciativa privada, es necesario fortalecer el comité de expertos, en la selección de personas idóneas y con un nivel académico alto en cada entidad estatal, y si no existiere crearlo, para que evalúe los proyectos de las APP en el país, y de ese modo evaluar de manera concienzuda los pros y los contras de aquel proyecto presentado por el sector privado para aceptar su favorabilidad o no.

Se debe llevar un control y vigilancia permanente por parte del Estado en las obras de infraestructura que se están desarrollando, para que cumplan con los estándares de calidad, tiempo, eficacia y servicio, y los sistemas de auditorías periódicamente puedan lograr detectar alguna falla en la ejecución y el funcionamiento de la obra y tomar las acciones correspondientes en el momento indicado.

\section{Referencias}

Agencia Nacional de Infraestructura. (s. f.). Vigencia futura. Recuperado de https://www. ani.gov.co/glosario/vigencia-futura

Alborta, G. R., Stevenson, C. y Triana, S. (2011). Asociaciones público-privadas para la prestación de servicios: una visión hacia el futuro. Banco Interamericano de Desarrollo. Recuperado de https://publications.iadb.org/en/asociaciones-publico-privadas-para-laprestacion-de-servicios-una-vision-hacia-el-futuro 
Akitoby, B., Hemming, R. y Schwartz, G. (2007). Inversión pública y asociaciones públicoprivadas. Washington, D. C., EE.UU.: International Monetary Fund. Recuperado de https://www.researchgate.net/profile/Gerd_Schwartz/publication/268396938_Inversion_publica_y_asociaciones_publico-privadas/links/54d0c6e20cf29ca811037192/ Inversion-publica-y-asociaciones-publico-privadas.pdf

Araujo, J. P. y Sierra, A. C. (2013). Concesiones de cuarta generación: impacto sobre los seguros de cumplimiento. Recuperado de http://www.fasecolda.com/files/7213/9101/0225/parte_i. captulo_4_concesiones_de_cuarta_generacin_e_impacto_sobre_los_seguros_de_cumplimiento.pdf

Baptiste Merizalde, S. (2013). Las asociaciones público-privadas en Colombia, reflejo teórico en la Ley 1508/2012, de 10 de enero. Recuperado de https://documentodegrado.uniandes. edu.co/documentos/Tesis_Santiago_Baptiste_Merizalde_13_mayo_de_2013.pdf

Caicedo Lara, D. (2010). El régimen de las concesiones y sus modalidades de contratación en Colombia. Bucaramanga, Colombia: SIC.

Constitución Política 1991, de 13 de junio, Constitución Política de 1991.

Contraloría General de la República. (2001). Evaluación de las concesiones viales en Colombia: una aproximación a sus pasivos contingentes. Bogotá, Colombia: Autor.

Córdoba Pumalpa, M. M. (2017). La implementación de la politica pública de infraestructura $y$ los mecanismos de las alianzas público-privadas. Los retos institucionales: caso Agencia Nacional de Infraestructura 2012-2016 (Tesis de maestría, Pontificia Universidad Javeriana, Bogotá, Colombia). Recuperado de https://repository.javeriana.edu.co/bitstream/ handle/10554/22295/DOCUMENTO.pdf?sequence=1\&isAllowed=y

Cortés Zambrano, S. P. (2013). La globalización económica y los derechos humanos. Via Inveniendi et Iudicandi, 8(2), 138-149. DOI: https://doi.org/10.15332/ s1909-0528.2013.0002.06

Decreto 222/1983, de 2 de febrero, por el cual se expiden normas sobre contratos de la nación y sus entidades descentralizadas y se dictan otras disposiciones. 
Decreto 2171/1992, de 30 de diciembre, por el cual se reestructura el Ministerio de Obras Publicas y Transporte como Ministerio de Transporte y se suprimen, fusionan y reestructuran entidades de la rama ejecutiva del orden nacional.

Decreto 2820/2010, de 5 de agosto, por el cual se reglamenta el Título VIII de la Ley 99 de 1993 sobre licencias ambientales.

Decreto 4165/2011, de 3 de noviembre, por el cual se cambia la naturaleza jurídica, cambia de denominación y se fijan otras disposiciones del Instituto Nacional de Concesiones (INCO).

Decreto 1467/2012, de 6 de julio, por el cual se reglamenta la Ley 1508/2012, de 10 de enero.

Decreto 1882/2012, 11 de septiembre, por el cual se adiciona el Presupuesto General de la Nación para la vigencia fiscal de 2012 y se efectúa la correspondiente liquidación.

Decreto 1082/2015, de 26 de mayo, por medio del cual se expide el Decreto Único Reglamentario del sector Administrativo de Planeación Nacional.

Decreto 431/2018, de 5 de marzo, por el cual se efectúa un ajuste en el Presupuesto General de la Nación para la vigencia fiscal 2018 y se efectúa la correspondiente liquidación.

Departamento Nacional de Planeación. (2018a). Contexto institucional para las APP en salud de Bogotá. Recuperado de http://www.saludcapital.gov.co/Documents/APP_2_P_Luis_ Fernando_Mejia.pdf

Departamento Nacional de Planeación. (2018b). Asociaciones público-privadas - APP—en infraestructura en Colombia. Bogotá, Colombia: Autor. https://www.medellin.gov.co/ $\mathrm{irj} / \mathrm{go} / \mathrm{km} /$ docs/wpccontent/Sites/Subportal\%20del\%20Ciudadano/Emprendimiento/ Secciones/Plantillas\%20Gen\%C3\%A9ricas/Documentos/2013/APP/DNP\%20-\%20 APP\%20en\%20Infraestructura\%20en\%20Colombia.pdf

Dinero. (2018, junio 19). Inició la licitación del Invías para la Ruta del Sol Sector II. Recuperado de: https://www.dinero.com/pais/articulo invias-abrio-licitacion-para-ruta-del-sol-sector-ii/259420 
Engel, E. M., Fischer, R. D. y Galetovic, A. (2014). Economía de las asociaciones públicoprivadas: una guía básica. Ciudad de México, México: Fondo de Cultura Económica.

Fuertes Fernández, A. (2007). Fundamentos de la colaboración público-privada para dotación de infraestructuras y servicios. REDETI: Revista de derecho de las telecomunicaciones e infraestructuras en red, 10(29), 37-124

Gómez Torres, D. y Ruiz Restrepo, M. A. (2015). Del contrato de concesión vial al contrato de APP por disponibilidad de la infraestructura y calidad del servicio en los proyectos de infraestructura vial en Colombia (Trabajo de investigación, Pontificia Universidad Javeriana, Bogotá, Colombia). Recuperado de https://repository.javeriana.edu.co/bitstream/ handle/10554/34516/GomezTorresDavidFelipe2015.pdf?sequence=1\&isAllowed=y

González Noriega, O. C. (2016). La omisión legislativa como hecho generador de la responsabilidad patrimonial del Estado. Verba Iuris, 35, 41-63.

Hernández Sandoval, R. A. (2016). Las asociaciones público-privadas, perspectivas y retos hacia el desarrollo de los principios de descentralización y asociatividad: favorecimiento al modelo privado en detrimento del modelo estatal (Tesis de maestría, Universidad Nacional de Colombia, Bogotá, Colombia).

Hernández Ucrós, M. M. (2014). Asociaciones público privadas: comentarios especiales a la Ley 1508/2012, de 10 de enero de la República de Colombia. Bogotá, Colombia: Universidad del Rosario.

Hernández Zorro, J. P. (2016). Contratos de asociación público-privada aplicación amplia o restrictiva en de la Ley 1508/2012, de 10 de enero: integración de lo público y lo privado (Tesis de maestría, Universidad Nacional de Colombia, Bogotá, Colombia).

Heros Echecopar, J. C. de los (2009). Asociaciones público-privadas: una mirada de cara al futuro. Revista de Derecho Administrativo, 7, 388-396.

HSBnoticias.com. (2015, octubre 22). Aprobada APP para renovación del Coliseo El Campin. Recuperado de http://hsbnoticias.com/noticias/bogota/ aprobada-APP-para-renovacion-del-coliseo-el-campin-164819 
Huertas Díaz, M. (2017). Asociaciones público-privadas de iniciativa privada de carácter social (Tesis de grado, Pontificia Universidad Javeriana, Bogotá, Colombia). Recuperado de https://repository.javeriana.edu.co/bitstream/handle/10554/34134/ HuertasD\%c3\%adazM\%c3\%b3nica2017.pdf?sequence=1\&isAllowed=y

Instituto Nacional de Vías. (2012, noviembre 20). Objetivos y funciones. Recuperado de https://www.invias.gov.co/index.php/informacion-institucional/objetivos-y-funciones

Ley 80/1993, de 28 de octubre, por la cual se expide el Estatuto General de Contratación de la Administración pública.

Ley 1150/2007, de 16 de julio, por medio de la cual se introducen medidas para la eficiencia y la transparencia en la Ley 80 de 1993 y se dictan otras disposiciones generales sobre la contratación con recursos públicos.

Ley 1508/2012, de 10 de enero, por la cual se establece el régimen jurídico de las asociaciones público privadas, se dictan normas orgánicas de presupuesto y se dictan otras disposiciones.

Ley 1753/2015, de 9 de junio, por la cual se expide el Plan Nacional de Desarrollo 20142018 "Todos por un nuevo país”.

Ley 1882/2018, de 15 de enero, por la cual se adicionan, modifican y dictan disposiciones orientadas a fortalecer la Contratación Pública en Colombia, la ley de infraestructura y se dictan otras disposiciones.

Leudo Paz, H. R. y Blanco Alvarado, R. C. (2016). Renegociación de contratos de concesión vial. Revista Republicana, 19, 71-192.

Loo Gutiérrez, M. (2015). Las asociaciones público-privadas en la Unión Europea: elementos para un análisis sobre la concesión de obra pública en Chile. Revista de Derecho, 45, 313-337. DOI: http://dx.doi.org/10.4067/S0718-68512015000200012

MAB Ingeniería de Valor S. A. (2016, noviembre 29). ¿̇ué son las concesiones viales de cuarta generación o $4 G$ ? Recuperado de http://mab.com.co/ que-son-concesiones-viales-de-cuarta-generacion- $4 \mathrm{~g} /$ 
Martínez Manzanedo, R. (2006). La contabilidad de la colaboración público-privada en el contexto del Sistema Europeo de Cuentas (SEC 95). Presupuesto y Gasto Público, 45, 187-198.

Mendoza Montoya, E. (2012). Asociaciones público-privadas: un análisis de marcos normativos en América Latina (Tesis de grado, Pontificia Universidad Javeriana, Bogotá, Colombia). Recuperado de https://repository.javeriana.edu.co/handle/10554/15382

Millán Durán, J., Blanco Alvarado, C. y Guecha Medina, C. N. (2017). Los procesos de selección de operadores privados del servicio público de televisión en torno a la libre concurrencia. En AA. VV., Justicia constitucional: Tomo II (pp. 200-220). Bogotá, Colombia: Ibáñez.

Ministerio del Interior. (2013, abril 10). Vigencia fiscal. Recuperado de https://www.mininterior.gov.co/content/vigencia-fiscal

Ministerio de Hacienda y Crédito Público. (s. f.). Asociaciones público-privadas (APPS) en entidades territoriales. Recuperado de http://www.minhacienda.gov.co/HomeMinhacienda/ShowProperty;jsessionid=shgA2xRkb797RG1Gf6n-zhrVKdYhyQz4F1rT11O nyIzkkkylw7Mh!1587083319?nodeId=\%2FOCS\%2FP_MHCP_WCC-064124\%2F\% 2FidcPrimaryFile\&revision=latestreleased

Moore, M. H. (2006). Creando valor público a través de asociaciones público-privadas. Trabajo presentado en X Congreso Internacional del CLAD sobre la Reforma del Estado y de la Administración Pública, Santiago de Chile, Chile.

Olave Méndez, L. M. (2016). Factores de infuencia en la expedición de la nueva regulación anticontrabando de 2015 en Colombia (Tesis de grado, Universidad del Rosario, Bogotá, Colombia). Recuperado de https://core.ac.uk/download/pdf/86437712.pdf

Pardo López, D. F. (2013). Análisis del bienestar de la implementación de alianzas públicoprivadas para la construcción y operación de espacios físicos para el desarrollo, en el sistema de atención integral de primera infancia del municipio de Santiago de Cali (Tesis de grado, Pontificia Universidad Javeriana, Bogotá, Colombia). Recuperado de https://repository. javeriana.edu.co/bitstream/handle/10554/12086/PardoLopezDiegoFernando2013. pdf?sequence $=1$ \&isAllowed $=y$ 
Pardo Posada, N. E. (2014). Un recorrido por los derechos colectivos en la jurisprudencia argentina. Via Inveniendi et Iudicandi, 9(1), 32-49. DOI: https://doi.org/10.15332/ s1909-0528.2014.0001.02

Moreno Velásquez, C. (2015). Asociaciones público-privadas en Colombia: ¿̇la calma después de la tormenta? (Trabajo de grado, Universidad de los Andes, Bogotá, Colombia). Recuperado de https://repositorio.uniandes.edu.co/bitstream/handle/1992/18696/u722235. pdf?sequence $=1$ \&isAllowed $=\mathrm{y}$

Quiroz, M. (2014). Acercamiento a las “oposiciones paradigmáticas” entre neoconstitucionalismo y positivismo jurídico. Revista IUSTA, 2(41), 77-97.

Reina Torres, A. L. (2015). Gobernanza y policy network para el estudio de las asociaciones público-privadas (APP) de infraestructura vial (Tesis de grado, Pontificia Universidad Javeriana, Bogotá, Colombia). Recuperado de https://repository.javeriana.edu.co/bitstream/ handle/10554/21670/ReinaTorresAngieLizeth2015.pdf?sequence=1\&isAllowed=y

Resolución 3656/2012, de 20 de diciembre, por la cual se establecen parámetros para la evaluación del mecanismo de asociación público-privada como una modalidad de ejecución de proyectos de que trata la Ley 1508/2012, de 10 de enero y el Decreto número 1467 de 2012.

Resolución 1464/2016, de 1 de abril, por la cual se establecen los requisitos y parámetros que deberán cumplir las entidades públicas responsables del desarrollo de proyectos de asociación público privada para solicitar el concepto previsto en el artículo 2016 de la Ley 1753/2015, de 9 de junio.

Rodríguez Cely, A. M. (2014). Indicadores de constitucionalidad de las políticas públicas: enfoque de gestión de derechos. Via Inveniendi et Iudicandi, 9(2), 135-175. DOI: https:// doi.org/10.15332/s1909-0528.2014.0002.06

Rodríguez Martínez, E. (2016). El pasaje del Estado y el derecho a la postmodernidad. Via Inveniendi et Iudicandi, 11(2), 11-37. DOI: https://doi.org/10.15332/ s1909-0528.2016.0002.01

Sentencia C-068/2009, de 10 de febrero, unidad normativa en demanda de inconstitucionalidad. 
Sentencia C-595/2014, de 20 de agosto, régimen jurídico de las asociaciones público-privadas y normas orgánicas de presupuesto.

Serrano Rodríguez, J. (2010). Financiamiento de infraestructura de transporte. Revista de Ingeniería, 32, 108-116.

Solarte Enríquez, P. (2013). Análisis y evaluación de las asociaciones público-privadas (APP) en Colombia (Tesis de grado, Universidad de los Andes, Bogotá, Colombia). Recuperado de https://repositorio.uniandes.edu.co/bitstream/handle/1992/12901/u703847. pdf?sequence $=1 \&$ isAllowed $=y$

Urueta, J. (2010). El contrato de concesión de obras públicas. Bogotá, Colombia: Ibáñez.

Vives, A. (2010). La responsabilidad social de las empresas: enfoques ante la crisis. Madrid, España: Fundación Carolina. Recuperado de https://www.euroamerica.org/pdf/RSEMexico_Libro.pdf

Wills Valderrama, S. (2011). Protección a la inversión extranjera en infraestructura por medio de Acuerdos Internacionales de Inversión: un nuevo reto para Colombia. International Law: Revista Colombiana de Derecho Internacional, 9(19), 17-38. Recuperado de https:// revistas.javeriana.edu.co/index.php/internationallaw/article/view/13733 